The Japanese Journal of Animal Psychology, 50, 1, 21-26 (2000)

Short Report

\title{
The number of trial upon the effects of US duration in conditioned licking suppression with rats
}

\author{
NOBUYUKI KAWAI ${ }^{1}$ \\ Primate Research Institute, Kyoto University, Japan
}

\begin{abstract}
Four groups of thirsty rats experienced Pavlovian conditioning during licking water. The experimental design was a factorial one. Two groups of rats received a short $(0.7 \mathrm{~s})$ shock as an unconditioned stimulus (US), while the other two groups received a longer shock ( $4.9 \mathrm{~s})$. The one of each shock duration group experienced a pairing of conditioned stimulus (CS) and US once in a session of 5 min long, while another of each experienced three pairings in a session of 5 min long. Despite of more pairings of CS-US in the three-trial groups than in the one-trial groups, conditioned suppression was more prominent in the one-trial groups than the three-trial groups. These results suggest that many CS-US pairings did not generate greater conditioned suppression, rather deteriorated it, which was accounted by the trials spacing effect. The baseline licking levels, which were regarded as reflecting the associative strength of the context with the US, were more suppressed in the three-trial groups than the one-trial groups. The conditioned suppression of the three-trial groups did not differ from each other, which is consistent with previous studies of US duration effect; Differences in US duration did not affect the level of conditioning under a between-subjects design. In contrast, the long US generated the greater conditioned suppression to the CS than the short US in the one-trial groups. These results suggest that the effects of US duration may be manifested under the condition, in which the associative value of the context is fairly weak.
\end{abstract}

Key words : US duration, number of trials, trial-spacing effect, conditioned suppression, rats.

In Pavlovian conditioning, there are many variables known to determine a final level of conditioning. For instance, a more intense unconditioned stimulus (US) produces a greater amount of conditioned response (CR) than a weaker US (e. g., Annau \& Kamin, 1961).

Manipulations of US duration also affect the level of conditioning, but the results are not straightforward. In some cases, the longer US, the greater CR evoked (e. g., Kawai \& Imada, 1996), while the opposite results were also obtained (e. g., Dufort, 1967; Runquist \& Spence, 1959). According to the summary by Kawai (1998; 1999), the long USs evoked the greater CRs than the short USs only when they were compared in a within-subjects design (Kawai, 1995, 1996; 1997; Kawai \& Imada, 1996, Exps. 3 \& 4; Tait, Kehoe, \&

1 Reprint request e-mail : nkawai@pri.kyoto-u.ac.jp
Gormezano, 1983, Exps. 2 \& 3). Many studies (e. g., Yamashita, Kawai, \& Imada, 1991) failed to find the effects of US duration under a between-subjects design.

However, there were a few exceptions; Only one trial conditioning in a session also seems to manifest the effects of US duration (i. e., a longer US elicites a greater $\mathrm{CR}$ ) even in a between-subjects design (Burkhardt \& Ayres, 1978; Kawai, 1995; Tait et al., 1983, Exp. 4). As Kawai (1999) pointed out, in case of one-trial procedure, the effects of US duration may not be weakened and/or blocked by a potentially more prominent context-US association.

One-trial procedure generates a longer inter-trial interval (ITI) than a procedure with more pairings in a session, when the session length is equated. According to the contextual-mediation models (Gibbon \& Balsam, 
1981; Miller \& Schachtman, 1985; Rescorla \& Wagner, 1972) of the trial spacing effect (TSE), a shorter ITI prevents a contextual extinction and hence a shorter ITI blocks conditioning of a CS (Rescorla \& Wagner, 1972). Such a weakened conditioning may obscure a potential difference in conditioning by different durations of US. In other words, the effects of US duration may be found when the context stimulus has a lower associative value by one trial pairing per session even under between-subjects comparison. Hence, the purpose of the present experiment is to study the development of conditioned suppression in rats by a short and a long duration of US, with one-trial or three-trial per session under a between-subjects comparison.

\section{Method}

\section{Subjects}

The subjects were 48 experimentally naive male rats of Wistar strain. Mean body weight was $246.4 \mathrm{~g}(248-323 \mathrm{~g})$ at the beginning of the experiment. The subjects were housed in stainless-steel wire cages in groups of two in a room maintained under on a 12:12 light-dark cycle. The light phase of the cycle started at 8:00 a. $m$. and the experimental session was run between 1:00 and 3:00 p. m. every day.

\section{Apparatus}

The apparatus consisted of six drinking boxes (cf. Kawai, Nishida, \& Imada, 1998), each having a transparent ceiling and white walls. Each drinking box had a grid floor and was $20 \mathrm{~cm}$ wide $\times 15 \mathrm{~cm}$ high $\times 10 \mathrm{~cm}$ deep (inside dimensions). In the lower part of the front wall of the box, there was a $12 \mathrm{~mm}$ diameter hole through which the rats could access to a drinking tube. When a rat made contact with the drinking tube, a current circuit between the tube and the grid floor of the box was closed, which activated a relay located in the next room. Each box was separated from the adjacent two by an equal distance on a table and the drinking-hole wall of each box faced the center of the table. A fan was attached directly below the center of the table to provide masking noise of approximately $64 \mathrm{~dB}$. The entire apparatus was illuminated during the experimental sessions with indirect light of approximately 6.7 lux into each box. A 10-s tone $(1000 \mathrm{~Hz}, 84 \mathrm{~dB})$ was delivered from a speaker suspended $200 \mathrm{~cm}$ directly above the center of the table. The $120 \mathrm{~V}$ ac $(0.43 \mathrm{~mA})$ electric shock of 0.7 $\mathrm{s}$ or $4.9 \mathrm{~s}$ duration was delivered to the grid floor through a $250-\mathrm{K}$ ohm current-limiting resistor in series with the rat.

\section{Procedure}

Prior to the start of the three phases of experiment, each rat was tamed by handling for five minutes per day for three consecutive days. After the handling on the third day, each rat was placed in $23.5 \mathrm{hr}$ water deprived schedule, which continued throughout this experiment. An experimental session was five min long.

Phase 1 (16 days): Preliminary training of drinking All subjects were trained in the drinking boxes for five min daily to drink water by licking the tip of the drinking tube. After each session the rats were returned to their home cages and, 10 min later, allowed to drink water for another 25 min. This preliminary training of drinking was continued for 16 days. Then, the rats were divided into four groups matched by the mean total number of licks and body weight, both on the last three days of Phase 1.

Phase 2 (14 days): Acquisition During the acquisition phase, Pavlovian conditioning trials were given in a daily session of $5 \mathrm{~min}$, superimposed upon licking response for 14 consecutive days. A conditioning trial consisted of $10 \mathrm{~s}$ presentation of a tone (CS), which was immediately followed by a $120 \mathrm{~V}$ ac $(0.43 \mathrm{~mA})$ footshock US. The experiment employed a $2 \times 2$ factorial design. One factor was the US duration $(0.7 \mathrm{~s}$ or $4.9 \mathrm{~s})$ and another was the number of trials per session (one or three). Group S-1 received the short US (0.7 s), given one CS-US pairing per session. Group L-1 also received one CS-US pairing per session, but it was done by the long US (4.9 s). Groups S-3 and L-3 experienced three CS-US pairings per session with the short and the long US, respectively. For Groups S-3 and L-3, the CSs were presented after $20 \mathrm{~s}, 160 \mathrm{~s}$, and $260 \mathrm{~s}$ from the beginnig of the session on the first day. They were $40 s^{-}-130 s^{-}-280 \mathrm{~s}, 30 \mathrm{~s}^{-}-110 \mathrm{~s}^{-}-250 \mathrm{~s}$, and $40 \mathrm{~s}-170 \mathrm{~s},-250 \mathrm{~s}$, on the second, third, and 
fourth day, respectively. For Groups S-1 and L-1, the trials started coincided with the second trial of the three trials groups. This series of sequences was repeated during the rest of sessions.

Phase 3 (8 days): Extinction The procedure was identical to that of Phase 2 except that no US was presented during this phase. All groups received eight sessions. As an index of conditioning, a conventional suppression ratio (SR, Annau \& Kamin, 1961) was used in the present experiment. The SR was calculated by B/ $(A+B)$, where B represents the number of licks during the $10 \mathrm{~s} C S$ and $\mathrm{A}$ the number of licks in $10 \mathrm{~s}$ immediately preceding the CS. In cases where A was zero, SR was regarded as zero.

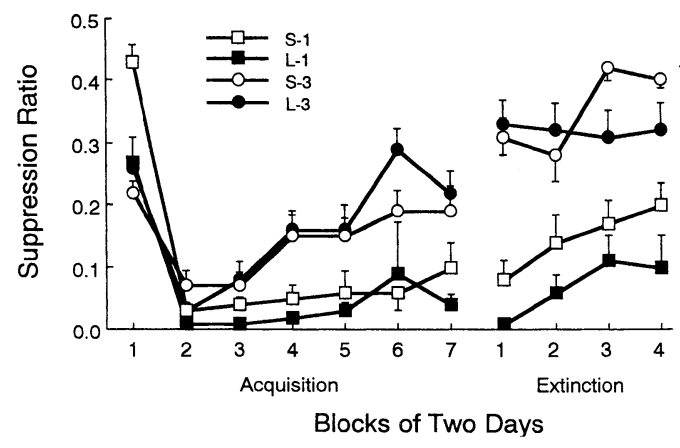

Figure 1. Mean suppression ratios of four groups in blocks of two days during acquisition (left panel) and extinction (right panel). The vertical lines mean S. E. M.

\section{Results}

Figure 1 shows the mean SRs in blocks of two days during acquisition and extinction phases for four groups. For each subject, a single SR was calculated for two successive days (pooling six trials for the three trials groups, two trials for the one trial groups). As shown in the left half of Figure 1, the acquisition was very rapid in all groups. As well as the other conditioned licking suppression studies (e. g., Kawai, 1996; Kawai \& Imada, 1996), the suppression was greatest at the second block in all groups, then it was gradually attenuated and became stable. In those processes, one trial groups showed greater suppression than the three trials groups, in spite of one third of pairings. There was no difference in suppression between three trials groups. These results are consistent with the previous studies which reported that different durations of US did not have a differential effect upon CRs in a between-subjects design (e. g., Kawai \& Imada, 1996, Exp. 2). However, Group L-1 showed greater suppression than Group S-1 except at the sixth block. An analysis of variance (ANOVA) of 2 (Duration) $\times 2$ (Number of trials) $\times 7$ (Block) was conducted for the acquisition data. The main effects of Number of trials $(F(1,39)=22.44$, $p<.001)$ and Block $(F(6,234)=57.59, p<.001)$ were significant, but the main effect of Duration was not significant. The interaction between Number of trials and Duration $(F(1,39)=6.59$, $p<.02)$ and that of Number of trials and Block $(F(6,234)=21.11, p<.001)$ were significant, the former suggesting the effect of US duration differed in the given number of pairings per session. The three-way interaction was also significant $(F(6,234)=2.66, p<.02)$, suggesting different rate of conditioned suppression among groups over blocks of trials. A subsequent Fisher's LSD test revealed that Group L-1 demonstrated signifincantly greater suppression than that of the other three groups, and Group S-1 was also suppressed more than Group L-3.

The mean licks during pre-CS periods of Phase 2 also differed among groups. They also varied as a function of sessions, but they were not systematic change, and there was no interaction with groups. Therefore, a single licks during pre-CS for each rat was averaged for 14 sessions. The mean licks were 49.2, 45.9, 44.6, and 34.4 for Groups S-1, L-1, S-3, and L-3, respectively. A 2 (Duration) $\times 2$ (Number of trials) ANOVA revealed the significant main effect of Number of trials $(F(1,44)=9.85, p<.005)$, and that of Duration $(F(1,44)=7.11, p<.02)$. These results were caused by the little responding in Group L-3. A Fisher's LSD test showed that Group L-3 differed from the other groups.

The right half of Figure 1 represents extinction data, where extinction is more dominant in three trials groups than one trial groups. The extinction process of three trials groups overlapped each other, however, Group L-1 showed consistently greater suppression than Group S-1. A 2 (Duration) $\times 2$ (Number of 
trials) $\times 4$ (Block) ANOVA was conducted on these data. The main effect of Number of trials $(F(1,44)=67.45, p<.001)$ and Block $(F(3,132)=$ $7.82, p<.001)$ were significant. The main effect of Duration was marginally significant $(F(1$, $44)=3.48, p<.07)$. There was no significant interaction effect.

The mean licks during pre-CS periods of Phase 3 were 52.6, 45.0, 45.4, and 44.2 for Groups S-1, L-1, S-3, and L-3, respectively. A 2 (Duration) $\times 2$ (Number of trials) $\times 4$ (Block) ANOVA revealed the main effect of Number of trials $(F(1$, $44)=4.41, p<.05)$, and Block $(F(3,132=3.34$, $p<.05)$. The effect of Duration $(F(1,44)=3.54$, $p<.07)$ was marginally significant. A subsequent LSD test revealed that Group S-1 responded much more than the other three groups during pre-CS periods in this phase.

\section{Discussion}

The results of the present study could be summarized as follows; 1 ) the conditioning was greater in the one trial groups than the three trials group, despite of greater frequency of pairings in the latter groups than that of the former groups, and 2) the effects of US duration (the longer US elicited the stronger CR than the shorter US) was manifested in the one trial groups, but not in the three trials groups.

These results seem to be partially consistent with the previous studies of US duration effects of Pavlovian conditioning. As clearly demonstrated (Kawai \& Imada, 1996) and repeatedly reported in many studies (e. g., Yamashita et al., 1991), the effects of US duration were not obtained in a between-subjects design experiment. However, as Kawai (1999) suggests, there were a few exceptions which found the effects of US duration in a between-subjects design (Burkhardt \& Ayres, 1978; a part of Kawai, 1995; Tait, et al., 1983, Exp. 4). It is important to mention that they presented, all in all, only one CS-US pairing in a session. Burkhardt and Ayres (1978) obtained the effects of US duration by just single CS-US pairing (i. e., the experiment was only one session). Therefore, the present study confirmed that the effects of US duration was manifested by a one trial procedure even if it is only one CS-US pairing (Burkhardt \& Ayres, 1978). No other study has investigated the effects of US duration in terms of the number of trials in a session.

Leaving a description of the results, how could we explain why the effects of US duration are effective under a one trial procedure? It seems well accounted by the difference of the associative strength of the context between one trial groups and three trials groups. Some models of Pavlovian conditioning (Gibbon \& Balsam, 1981; Miller \& Schachtman, 1985; Rescorla \& Wagner, 1972) assume that a longer ITI evokes a greater CR than a short ITI, because a long ITI promotes a greater contextual extinction (e. g., Barela, 1999; Kawai, 1996; Papini \& Brewer, 1994). In other words, a short ITI would block conditioning of a CS (Rescorla \& Wagner, 1972) or prevent a response modulation to a CS (Miller \& Schachman, 1985; Yin et al., 1994) with a stronger associative strength of context.

As predicted by these models, pre-CS levels (the baseline), which were assumed to reflect the associative strength of the context, were lower in short ITI groups (i. e., Groups S-3 and L-3 in this study), although this might just reflect the difference in aversiveness caused by many USs. Groups L-3 experienced most severe condition, which might suppress any responding during session (cf. Kawai \& Imada, 1990). In any case, the strong associative value of the context made the CRs of the three trials groups (Groups S-3 and L-3) weaker than that of one trial groups. Such strong associative values of context in Groups S-3 and L-3 also might diminish a slight or potential difference of CRs in the three trials groups, which might have emerged under a weaker associative strength of the context. Remember that only Group L-3 showed the greater pre-CS (assumed as baseline level) suppression than the other three groups in the acquisition phase. This made the SR of Group L-3 higher, because the fewer responding during pre-CS periods makes a denominator for the SR small. However, there was no difference in the pre-CS periods between Groups S-1 and L-1. Thus, the weak effects of US duration might manifest in conditioned suppression under weak and/or equalized associative values of the context. This prediction 
is consistent with Kawai (1996), in which the differential conditioning of US duration was greater under the long ITI than that of the short ITI. Yamashita et al. (1991) also reported that the baseline responses were suppressed as a function of US duration, but the effects of US duration could not be found in the same experimental situation as the present study.

As well as a differential conditioning (Kawai, 1995; 1996; 1997), one trial procedure could be a reliable situation to manifest the effects of US duration, by weakening (Tait et al., 1983, Exp. 4) or equalizing (Tait, et al., 1983, Exps. $2 \& 3$ ) the context associative strength among groups (Kawai, 1999). Further researches, which explore the role of the contextual associative value upon the effects of US duration, will be required.

\section{References}

Annau, Z., \& Kamin, L. J. 1961 The conditioned emotional response as a function of the intensity of the US. Journal of Comparative and Physiological Psychology, 54, 428-432.

Barela, P. B. 1999 Theoritical mechanisms underlying the trial-spacing effect in Pavlovian fear conditioning. Journal of Experimental Psychology: Animal Behavior Processes, 25, 177-193.

Burkhardt, P. L., \& Ayres, J. J. B. 1978 CS and US duration effects in one-trial simultaneous fear conditioning as assessed by conditioned suppression of licking in rats. Animal Learning \& Behavior, 6, 225-230.

Dufort, R. H. 1967 Eyelid conditioning as a function of US duration with drive equated. Journal of Experimental Psychology, 74, 289294.

Gibbon, J., \& Balsam, P. D. 1981 Spreading association in time. In C. M. Locurto, H. S. Terrace, \& J. Gibbon (Eds.), Autoshaping and conditioning theory. NY: Academic Press. Pp. 255-284.

Kawai, N. 1995 Simultaneous contrast effects in a classical aversive conditioning situation with rats, Japanese Journal of Research on Emotion, 3, 17-25.

Kawai, N. 1996 Differential conditioning of US duration in rats: The effects of inter- transitional interval, Japanese Journal of Psychology, 67, 396-402.

Kawai, N. 1997 The effects of US intensity on the differential conditioning of US duration in rats, Japanese Journal of Animal Psychology, 47, 59-66.

Kawai, N. 1998 A behavioral approach for the brain function of a discrimination: The stimulus arrangements enhance the discrimination, Behavioral Science Research, 37, 55-66.

Kawai, N. 1999 The role of US duration in classical conditioning, Japanese Psychological Review, 42, 86-103.

Kawai, N., \& Imada, H. 1990 Attenuation of conditioned suppression by immediately preceding primary and secondary aversive stimuli in rats, Japanese Journal of Animal Psychology, 40, 20-28.

Kawai, N., \& Imada, H. 1996 Between- and within-subject effects of US duration on conditioned suppression in rats: Contrast makes otherwise unnoticed duration dimension stand out. Learning and Motivation, 27, 1-20.

Kawai, N., Nishida, N., \& Imada, H. 1998 Effects of postconditioning manipulations following compound conditioning on conditioned licking suppression in rats. Psychologia, 41, 49-59.

Miller, R. R., \& Schachtman, T. R. 1985 Conditioning context as an associative baseline: Implications for response generation and the nature of conditioned inhibition. In R. R. Miller \& N.E. Spear (Eds.), Information processing in animals: Conditioned inhibition. Hillsdale, NJ: Erlbaum. Pp.51-88.

Papini, M. R., \& Brewer, M. 1994 Response competition and the trial-spacing effect in autoshaping with rats. Learning and Motivation, 25, 201-215.

Rescorla, R. A., \& Wagner, A. R. 1972 A theory of Pavlovian conditioning: Variations in the effectiveness of reinforcement and nonreinforcement. In A. H. Black \& W. F. Prokasy (Eds.), Classical conditioning II. Current theory and research. NY: Appleton-Century-Crofts. Pp.64-99. 
Runquist, W. N., \& Spence, K. W. 1959 Performance in eyelid conditioning as a function of UCS duration. Journal of Experimental Psychology, 57, 249-252.

Tait, R. W., Kehoe, E. J., \& Gormezano, I. 1983 Effects of US duration on classical conditioning of the rabbit's nictitating membrane response. Journal of Experimental Psychology: Animal Behavior Processes, 9, 91101.

Yamashita, H., Kawai, N., \& Imada, H. 1991, The effect of US duration on licking conditioned suppression, Jinbunronkyu 41, 63-74.

Yin, H., Barnet, R. C., \& Miller, R. R. 1994 Trial spacing and trial distribution effects in Pavlovian conditioning: Contributions of a comparator mechanism. Journal of Experimental Psychology: Animal Behavior Processes, 20, 123-134.

This experiment was conducted in Kwansei Gakuin University.

(Received Oct. 1, 1999 ; accepted Jan. 14, 2000) 